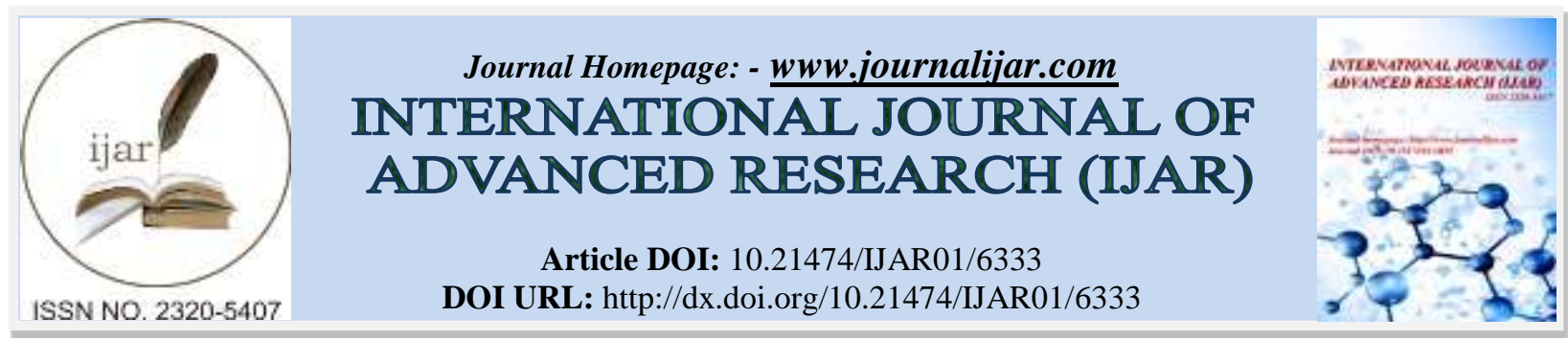

RESEARCH ARTICLE

\title{
SUPERIOR LIMIT OF THE THERMOVOTOVOLTAIC EFFICIENCY SOLAR CELLS IN NEAR FIELD AND FAR FIELD: COMPARATIVE STUDY.
}

\author{
Waly DIALLO*, Saliou NDIAYE, Mamadou NIANE, Omar. A. NIASSE and Bassirou BA. \\ Laboratoire de semi-conducteurs et d'énergie solaire, Département de physique, Faculté des sciences et techniques \\ (UCAD-SENEGAL).
}

\section{Manuscript Info}

Manuscript History

Received: 17 November 2017

Final Accepted: 19 December 2017

Published: January 2018

Key words:-

TPV, far-field, near-field, conversion, energy.

\section{Abstract}

Thermophotovoltaic conversion (TPV) is very coveted nowadays because of its output and its high density of power. Its literature is relatively young and only since 1961 in the context from the cold war between the two super power dates. Carried out work enabled us to see the limiting outputs until one can wait in this energy transformation as well into near field into far field. The best performance obtained in far field turns around $43 \%$ exceeding the thermodynamic output predicted by Schockley-Queisser (33\%) and which is in net agreement with the output of Carnot. It during the outputs obtained for an ideal system is higher than those of Carnot thus utopian. The limiting outputs for a system TPV in near field give results largely higher than those of the far field. What is satisfying it is that they are not exult by the output of Carnot thus realizable.

Copy Right, IJAR, 2018,. All rights reserved.

\section{Introduction:-}

The devices thermophotovoltaic (TPV) are conversion systems of energy which generate the electric power directly starting from a radiation thermic. There are nowadays several approaches which try to exploit this die which offers convincing results exceeding even those of the photovoltaic system to silicon. Among these approaches we can quote the electromagnetic approach (when it is about the mode in far field) [1, 2]; approach of the electrodynamics fluctuationnel (when it is about the near field) [3-5]. Its output is limited in theory by the thermodynamic limit of Schockley-Queisser which corresponds if the source is a black body and its value turns around 33\% [1]. This limit can be easily overcome nowadays with a monochromatic source when the energy received by cell TPV coincides with the energy of gap of the semiconductor or while reducing outdistances it which separates the source with a value lower than the wavelength from emission.

Work that we propose is to see the limits higher than one can await these two conversion systems and to make a comparative analysis of it.

\section{Principle Of Operation Of A Thermophotovoltaic Device:- In the far field:-}

The classical theory of the thermal radiation, developed by Planck, is based on the concept of black body [6-8]. It is an ideal transmitter which emits more energy than any other body with any wavelength. The radiation emitted by the black body is independent of the matter of which it is made up. The thermal radiation of the black body of Planck however is limited to a fundamental assumption: the distances between the bodies in exchange are higher than the dominant wavelength of the emission [2], so that thermal energy is transferred through the progressives waves only. 
Thermophotovoltaic conversion is divided into two parts according to the type of radiation considered. There is the TPV in far field which takes as a starting point the thermal radiation black or of any emitting body of heat with a wavelength lower than the distance which separates cell statement and the thermal source [9].

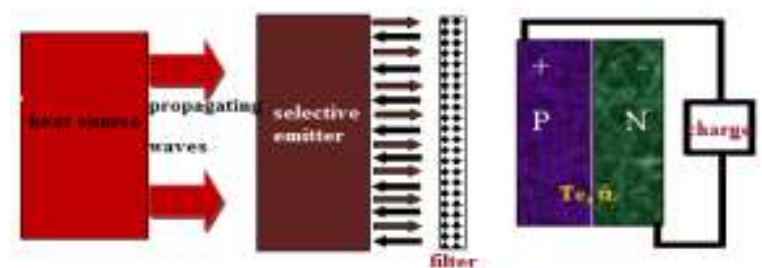

Fig. 1:- diagram of a system TPV which functions in far field

\section{In Near Field:-}

Contrary to the first, these two parts are separated from a distance lower than the wavelength. The thermal source emits waves evanescence's which are confined very close to the surface of the body [1, 2], which does not carry importance in calculations of the far field. These waves evanescence's exist and are propagated along the interface between two materials, while decreasing exponentially on a distance approximately of a wavelength of the normal of this interface.

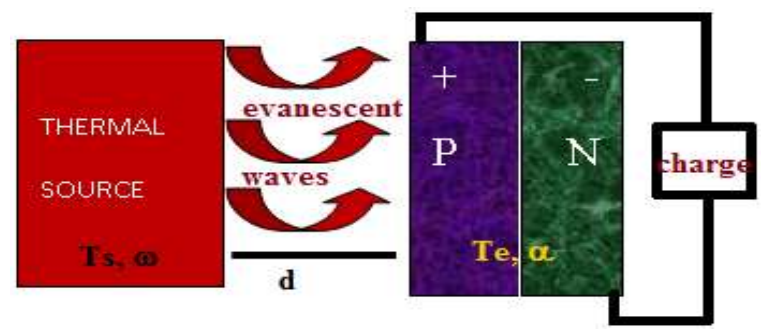

Fig. 2:- Diagram Of A System Tpv Which Functions In Near Field

If the source emits in open space, flow Net of energy of the field evanescent is consequently null. If open space separating the two parts is narrowed at a distance lower than the wavelength so that its surface is in the field evanescent, the movement loads in the cell is affected by the presence of this near field. The resulting electronic movement dissipates the energy of the near field in the cell by Joule effect. This phenomenon is called tunnel effect of radiation, and can be seen as an additional channel in which radiant energy can run out, involving the going beyond of the transfer predicted by the distribution of black body of Planck.

\section{Efficiency Tpv:-}

Efficiency conversion in far field:-

The efficiency conversion is expressed as follows:

$$
\eta_{T P V}=\frac{P_{e l}}{P_{r a d}}
$$

To make a comparative study of the higher limit of the two types of efficiency conversion, we have: $u=\frac{h \omega}{2 \pi \cdot K_{B} T_{S}}$

$\omega$ is the frequency of emission, $T_{S}$ is the temperature of the source and $\mathrm{h}$ is the Planck's constant. The maximum Power of a body emitting in the far field is [1]:

Error! 
$\lambda_{\mathrm{G}}$ is the length corresponding to the forbidden band of material of cell statement, $A_{C}$ is the surface of the cell; $q_{i c}$ : the flow of incidental radiation on cell statement per unit of length of wave, $e_{b}$ is the power of emission of the black body which the transmitter $\rho_{\mathrm{c}}$ constitutes is the reflectance of the cell; the radiative power's [10], :

$$
P_{\text {rad }}=\frac{\left(K_{B} T_{S}\right)^{4}}{4 \pi^{2} \hbar^{2} c^{2}} \int_{0}^{\infty} \frac{u^{3}}{e^{u}-1} d u
$$

\section{Efficiency conversion in nigh field:-}

The power generated in the photodiode is calculated as being the product of the tension $\mathrm{V}_{0}$, e the electron charge and the difference between flow of absorptive photons and flow of re-emitted photons [3].

$$
\begin{aligned}
& P_{P V}=\frac{1}{\pi^{2}} \int_{0}^{\infty} d q q\left[\begin{array}{l}
\int_{\omega_{g}}^{\infty} d \omega \Pi(\omega, q) \frac{e V}{e^{\left.\frac{\hbar \omega}{k T e}\right)}-1} \\
-\int_{\omega_{g}}^{\infty} d \omega \Pi(\omega, q) \frac{e V_{0}}{e^{\frac{\hbar \omega-e V_{0}}{k T_{S}}}-1}
\end{array}\right] \\
& \Pi(\omega, q)=\frac{\operatorname{Im}\left(\frac{\varepsilon_{1}-1}{\varepsilon_{1}+1}\right) \operatorname{Im}\left(\frac{\varepsilon_{2}-1}{\varepsilon 2+1}\right)}{\left|1-\left(\frac{\varepsilon_{1}-1}{\varepsilon_{1}+1}\right)\left(\frac{\varepsilon_{2}-1}{\varepsilon_{2}+1}\right) e^{-2 q d}\right|^{2}} e^{-2 q d}
\end{aligned}
$$

$\mathrm{V}_{0}$ the potential difference to which the cell is operative, $\mathrm{q}$ is the component parallel of the vector of wave, $\varepsilon_{1}$ and $\varepsilon_{2}$ are the functions dielectric the transmitter and of the cell respectively; $\mathrm{d}$ is the distance separating the source and the cell; $\square_{\mathrm{g}}$ is the frequency of the gap of the cell.

$$
\mathrm{V}_{\max 0}=\omega_{\mathrm{g}}\left(1-\frac{\mathrm{T}_{\mathrm{S}}}{\mathrm{T}_{\mathrm{e}}}\right)
$$

By modelling cell statement like a thermodynamic diode ideal operating with the voltage $\mathrm{V}_{0}$, we can write the radiative power of exchange as follows [10]:

$$
P_{r a d}=\frac{1}{\pi^{2}} \int_{0}^{\infty} d q q\left[\begin{array}{c}
d \omega \Pi(\omega, q) \frac{\hbar \omega}{e_{0}^{\infty}} \\
-\int_{\omega_{g}}^{\infty} d \omega \Pi(\omega, q) \frac{\hbar \omega}{e^{\frac{\hbar \omega-e V_{0}}{k T_{S}}}-1}
\end{array}\right]
$$

\section{Results and Discussion:-}

\section{Ultimate performance in far field:-}

The maximum output is obtained by considering that the transmitter is opaque so that the flux density of the transmitter is equal to the absorptance which we can have $\varepsilon_{E}=1-\rho_{c}$ that being so would like to say that the transmitter re-emits all the energy which it received from the heat source. In addition to these considerations having to us to consider constant reflectance. The maximum output will be thus expressed: 


$$
\eta_{\max } \approx \frac{15}{\pi^{4}} u_{g}\left[u_{g}^{2}+2 u_{g}+2\right] e^{-u_{g}}
$$

The value of $\mathrm{u}_{\mathrm{G}}$ which gives the greatest output of conversion thermophotovoltaic is 2 .

We thus need a weak material of gap receiving a reasonable temperature; what is confirmed.

\begin{tabular}{|c|c|c|c|c|}
\hline Semiconductor & $\begin{array}{c}\text { Gap Energy } \\
(\mathrm{eV})\end{array}$ & $\begin{array}{c}\text { Source température } \\
(\mathrm{K})\end{array}$ & $\mathrm{u}_{\mathrm{g}}$ & $\begin{array}{l}\text { Maximal } \\
\text { efficiency }\end{array}$ \\
\hline $\mathrm{Si}$ & 1,1 & 1200 & 10,626 & 0,54 \\
\hline InGaAsP & 6,76 & 1200 & 6,76 & 7,38 \\
\hline InGaAs & 0,6 & 1200 & 5,794 & 12,81 \\
\hline InGaAsSb & 0,53 & 1200 & 5,118 & 18,13 \\
\hline InPAsSP & 0,5 & 1200 & 4,829 & 20,79 \\
\hline GaInAsPSb & 0,35 & 1200 & 3,38 & 35,76 \\
\hline
\end{tabular}

Table 1:- maximum value of output TPV in far field of some materials

Table 2:- maximal values of TPV efficiency in far field of some devices

\begin{tabular}{|c|c|}
\hline Semi-conducteur & efficiency (\%) \\
\hline $\mathrm{Si}$ & 90 \\
\hline InGaAsP & 84 \\
\hline InGaAs & 81.3 \\
\hline InGaAsSb & 78.9 \\
\hline InPAsSP & 77.6 \\
\hline GaInAsPSb & 68.8 \\
\hline
\end{tabular}

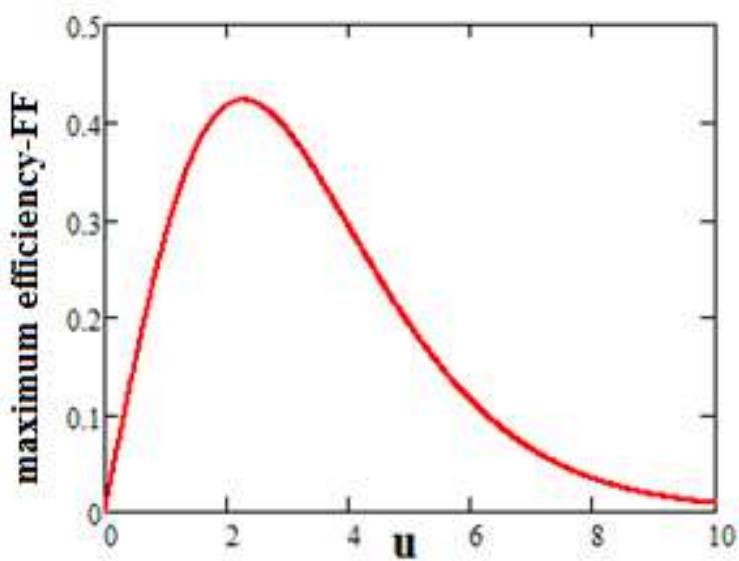

Fig. 3:- maximum efficiency TPV in far field

\section{Ideal performance in far field:-}

The preceding considerations do not hold counts of them energies of the inferior photons to that of the gap of the cell which can taken part in the harmful effect of the cell and the reduction of the output. For an ideal output, the transmitter should not receive energies of the inferior gap to the cell. For that, the flux density must be null and reflectance equal to the unit. As for photons whose energies are higher than that of the gap, emittance must be equal to the unit and null reflectance. The expression of the output becomes: 


$$
\eta_{\max }=u_{g} \frac{\int_{u_{g}}^{\infty} \frac{u^{2}}{e^{u}-1} d u}{\int_{u_{g}}^{\infty} \frac{u^{3}}{e^{u}-1} d u}
$$

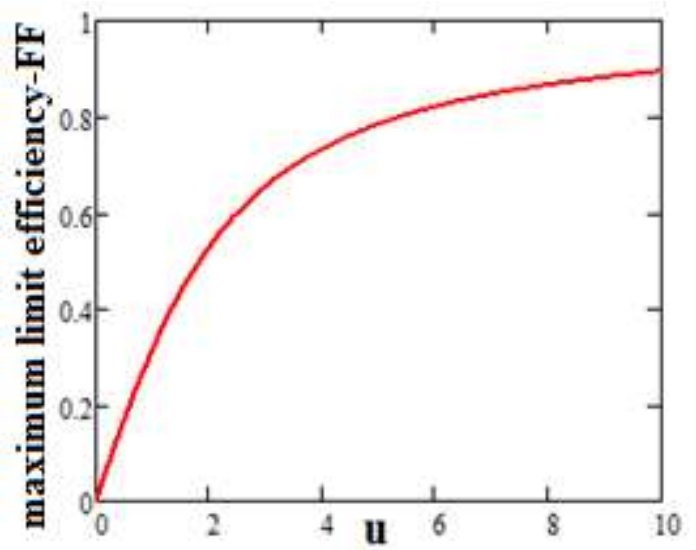

Fig. 4:- efficiency TPV of ideal system in far field.

\section{Maximun limit efficiency TPV in near field:-}

We treat here the higher limit of the output for a thermal radiation in near field which is similar to the near relationmonochromatic radiation [11-12]. This radiation is largely dominated by the frequency of the mode of resonance this is why this output corresponds to that of a quasi-monochromatic source.

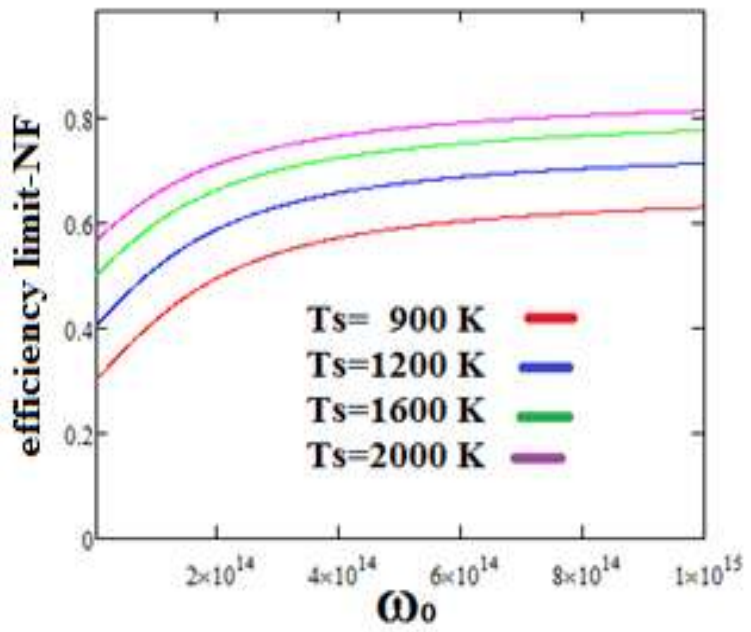

Fig.5:- higher limit of output TPV in near field according to the frequency mode of resonance

We have the increase efficiency in when the frequency of resonance increases but also when the temperature increases. This is seen moreover meadows while posing:

$$
u=\frac{h \omega_{0}}{2 \pi \cdot K_{B} T_{S}}
$$

It is seen here that between 0 and 2, the output falls before taking an ascending form like illustrates it the Fig 6 . 


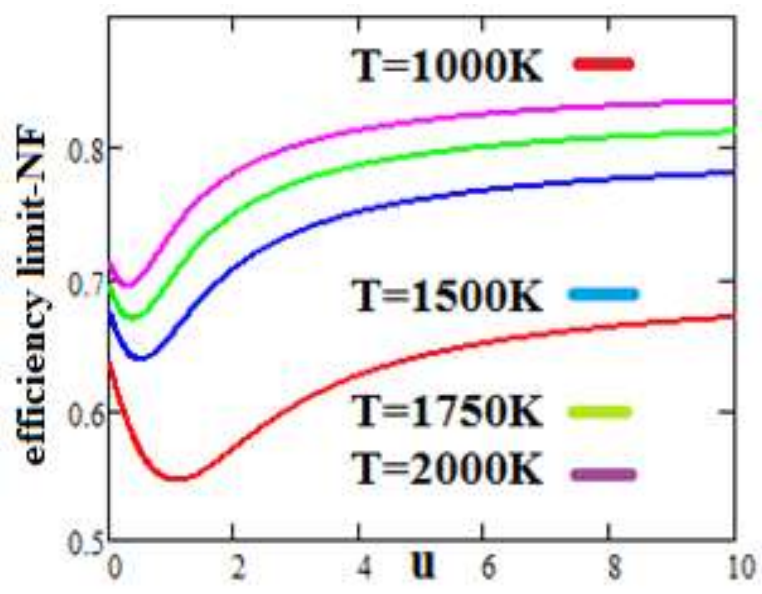

Fig.6:- higher limit of output TPV in close field according to $u$.

\section{Comparaison of limit efficiency conversion in far and near field :-}

Fig7: comparison of the efficiencies of two conversion types.

We saw previously and separately the limiting output of these two types of conversion TPV. At present, we go compared. The figure (a) shows us that the minimal value of the higher ultra-limit of the output of a device TPV functioning in near field with a temperature to $1500 \mathrm{k}$ is largely higher than the maximum value of the maximum expression of the output in far field.
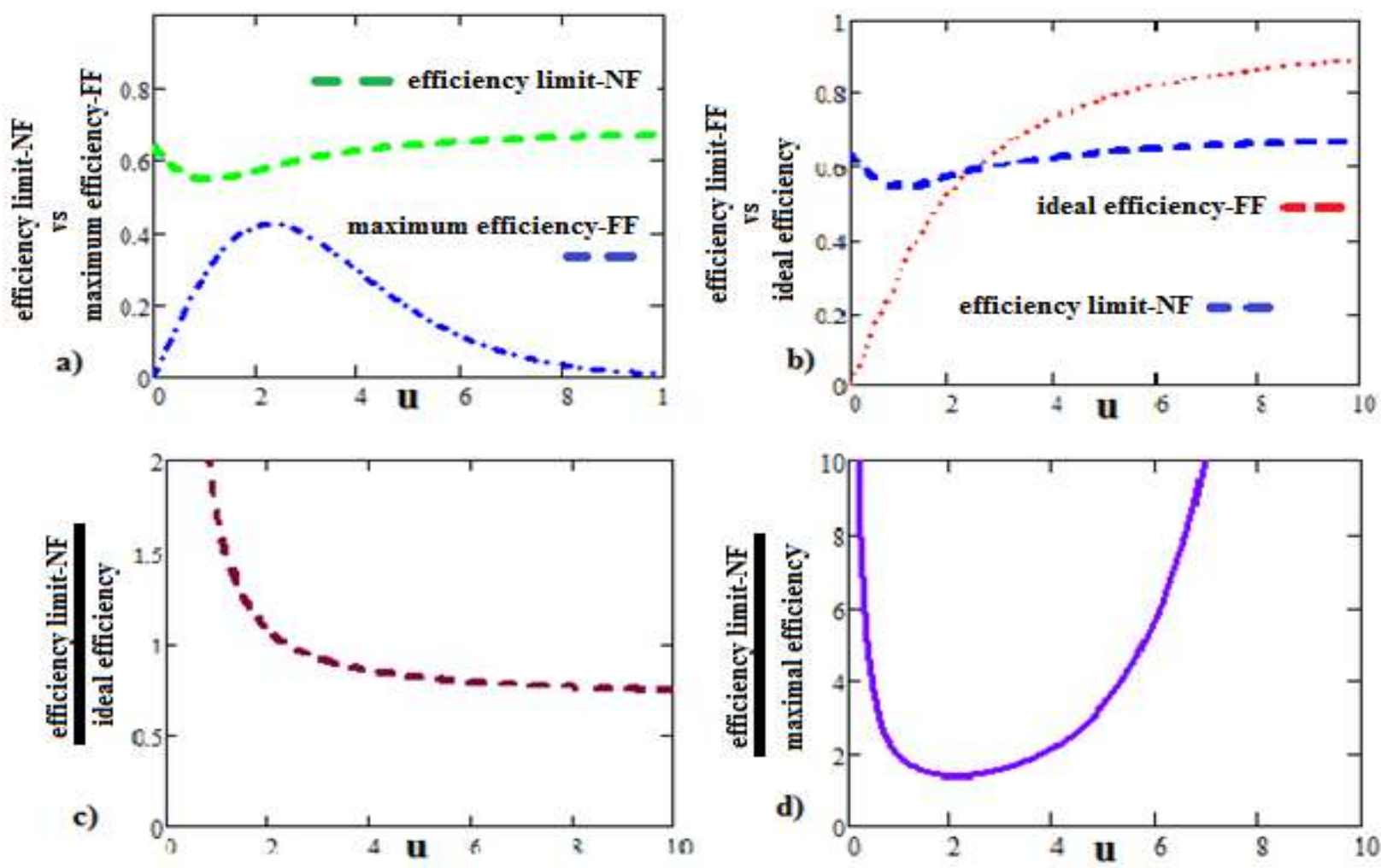

The figure (b) shows us that a device TPV ideal which functions in far field can have an output superior with that which function in the near field by having a material which can give u high. For an apparatus TPV containing silicon $\left(\mathrm{E}_{\mathrm{g}}=1.1 \mathrm{eV}\right)$ whose temperature of emission is of $1200 \mathrm{~K}$, the awaited ideal output is $90 \%$. The output of Carnot for a cell whose temperature of emission is of 300k raises to $75 \%$. That seems utopian then for this device. As for the apparatus functioning in far field, the output envisaged is $72.6 \%$. 
The course (c) is the report/ratio of the output of the higher limit near field and of the output limited ideal in far field while the curve d) translates the report/ratio of the output of conversion of the near field and the maximum far field.

\section{Conclusion:-}

We saw that conversion TPV offers a convincing output of conversion being able to exceed the limiting value envisaged by Schockley-queisser; that it is of the near field or the far field. Of these two types of conversion TPV, one sees that the limit of the near field is more promising. This limit is in perfect agreement with the limiting output of Carnot; what is not the case with ideal output TPV in far field.

\section{Reference:-}

1. Donald L. Chubb, Fundamentals of Thermo photovoltaic Energy Conversion, Elsevier B.V; Amsterdam, 2007.

2. B.Thomas, thermophotovoltaics basic principles and critical aspects of system design, Allemagne: Springer, 2011.

3. P. Keunhanet Z. Zhang, Fundamentals and applications of near-field radiative energy transfer, Global Digital Central, vol. 4, 11.3001, 2013.

4. Mathieu Francoeur, Near-field radiative transfer: thermal radiation, thermophotovoltaic power generation and optical characterization, University of Kentucky UK, 2010.

5. Daneshvar, H.; Prinja, R.; Kherani, N.P. Thermophotovoltaics: Fundamentals, challenges and prospects. Appl. Energy, 159, 560-575, 2015

6. E.V. Kunitsyna et al,Narrow gap III-V materials for infrared photodiodes and thermophotovoltaic cells, Optical Materials, Elsevier ; Volume 32 ; Pages 1573-1577 ; 2010.

7. Mohannad T. Aljarrah, modeling and experimental validation of radiative heat transfer in porous nanocomposites as selective emitters for low temperature thermophotovoltaic systems, Faculty of The University of Akron, 2009 .

8. Berkaï Zakaria, Etude et simulation d'une cellule thermo-photovoltaïque, mémoire, UNIVERSITE DE BECHAR Faculté des Sciences et Technologie, 2012

9. Waly Diallo , Nacire Mbengue, Moulaye Diagne, Cheikh Mbow, Bassirou BA, Ultras TPV Conversion Efficiencies Depending on the Energy Gap of The Materials Used and the Source Emission Temperature, Current Trends in Technology and Science, : 2279-0535. Volume : 04, 03, 2015

10. M. Laroche, Role des ondes de surface dans la modification des proprietés radiative de materiaux microstructurés. Application à la conception de sources infrarouges et à l'effet thermophotovoltaique, Paris: Ecole Centrale Paris, 2006.

11. L. Ivan, P.-M. Agustin et M. R. J., Thermodynamics and energy conversion of near-field thermal radiation: Maximum work efficiency bounds, EDP Sciences, vol. 79, 101001, 2014.

12. N.Arvind et Z. Yi, Theory of thermal nonequilibrium entropy in near-field thermal radiation, PHYSICAL REWIEW, vol. 88, 1075412; 2014. 